\title{
MAGNETIC MULTILAYER INTERFACE ANISOTROPY
}

\author{
TECHNICAL PROGRESS REPORT \\ January 1, 1992 through December 31, 1992 \\ Submitted to The USDOE Office of Basic Energy Sciences \\ Grant Number DE'-FG02-86ER45281
}

\author{
By Michael J. Pechan \\ Miami Usiversity
}

\section{DISCLAIMER}

\begin{abstract}
This report was prepared as an account of work sponsored by an agency of the United States Government. Neither the United States Government nor any agency thereof, nor any of their employees, makes any warranty, express or implied, or assumes any legal liability or responsibility for the accuracy, completeness, or usefulness of any information, apparatus, product, or process disclosed, or represents that its use would not infringe privately owned rights. Reference herein to any specific commercial product, process, or service by trade name, trademark, manufacturer, or otherivise does not necessarily constitute or imply its endorsement, recommendation, or favoring by the United States Government or any agency thereof. The views and opinions of authors expressed herein do not necessarily state or reflect those of the United States Government or any agency thereof.
\end{abstract}

\section{MASTER}




\title{
MAGNETIC MULTILAYER INTERFACE ANISOTROPY
}

\begin{abstract}
$\mathrm{Ni} / \mathrm{Mo}$ and $\mathrm{Ni} / \mathrm{V}$ multilayer magnetic anisotropy has been investigated as a function of $\mathrm{Ni}$ layer thickness, frequency and temperature. Variable frequency ferromagnetic resonance (FMR) measurements show, for the first time, significant frequency dependence associated with the multilayer magnetic anisotropy. The thickness dependence allows one to extract the interface contribution from the total anisotropy. Temperature dependent FMR ( $9 \mathrm{GHz})$ and room temperature magnetization indicate that strain between $\mathrm{Ni}$ and the non-magnetic layers is contributing significantly to the source of the interface anisotropy and the state of the interfacial magnetization. In order to examine the interface properties of other transition metal multilayer systems, investigations on $\mathrm{Fe} / \mathrm{Cu}$ are underway and $\mathrm{CoCr} / \mathrm{Ag}$ is being proposed.

ESR measurements have been reported on $\mathrm{Gd}$ substituted $\mathrm{YBaCuO}$ superconductors and a novel quasi-equilibrium method has been developed to determine quickly and precisely the transition temperature.

During the next project period the P.I. proposes to (1) extend the variable frequency FMR measurements to low temperature, where extremely large interface anisotropies are known to obtain in $\mathrm{Ni} / \mathrm{Mo}$ and $\mathrm{Ni} / \mathrm{V}$ and are proposed to exist in $\mathrm{Ni} / \mathrm{W}$; (2) obtain accurate dc anisotropies via a novel, variable temperature torque magnetometer currently under construction; (3) expand upon his initial findings in $\mathrm{Fe} / \mathrm{Cu}$ multilayer investigations; (4) begin anisotropy investigations on $\mathrm{Co} / \mathrm{Ag}$ and $\mathrm{CoCr} / \mathrm{Ag}$ multilayers where the easy magnetization direction depends upon the $\mathrm{Cr}$ concentration; (4) make and characterize $\mathrm{Bi}$ based superconductors according to resistivity, thermal conductivity and thermoelectric power and construct YBaCuO based superconducting "loop-gap" resonators for use in his magnetic resonance work.
\end{abstract}


The past year our efforts were prixnarily concentrated on 1) characterizing anomalous ferromagnetic resonance (FMR) modes in interlayer coupled magnetic multilayered systems; 2) refinement of our low temperature torque measurement capabilities and design of an in situ transducer; and 3) improving the state of torque data analysis techniques. A novel design for controlling the frequency of a FMR spectrometer was built, tested and published. And thermal conductivity of high temperature superconductor samples was measured as part of an undergraduate research project.

\section{Fe Based Multilayers}

Effects of dynamic interlayer coupling have been investigated utilizing samples where the nonmagnetic layer thickness is varied while holding the $\mathrm{Fe}$ layer thickness constant. Representative ferromagnetic resonance (FMR) spectra from $\mathrm{Fe}_{30} \AA / \mathrm{Cr}_{\mathrm{x}}$ and $\mathrm{Fe}_{50} \AA \mathrm{Cu}_{\mathrm{x}}$ multilayer samples, where the applied field is oriented parallel to the film plane, are shown in Fig. 1.

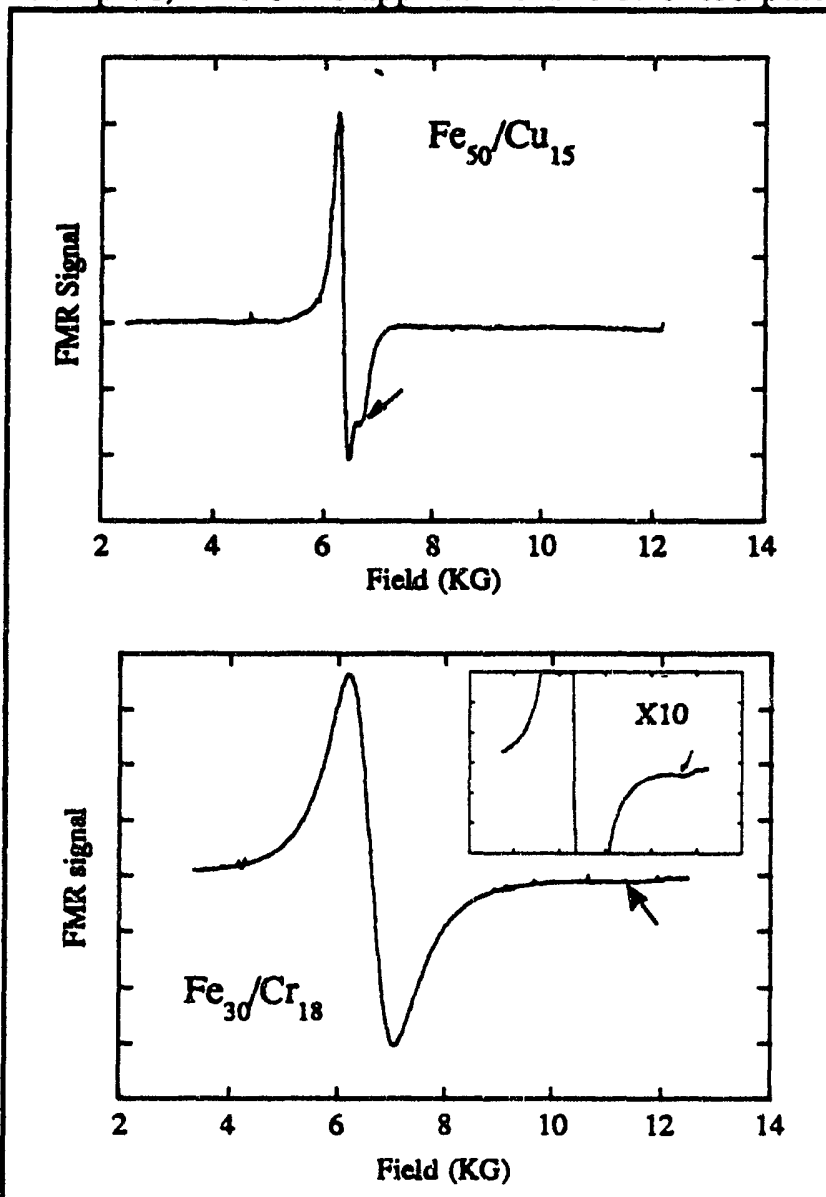

Particularly noteworthy is the "anomalous" mode which appears in addition to the large mode (produced by the magnetization's uniform precession in the applied field). The appearance and structure of such anomalous modes are observed to depend upon nonmagnetic layer thickness in these systems. We suspect that these modes arise from interlayer coupling effects and have developed a model to test our hypothesis. The assumption is made that the Fe intralayer coupling is much greater than the interlayer coupling, $\mathrm{J}$, and as a result the $\mathrm{Fe}$ layers will respond dynamically like rigid, coupled rotators. The coupling energy between $\mathrm{Fe}$ moments directly across the nonmagnetic layer is given by

$$
U=2 J N_{m}^{2} \hat{\mu} \cdot \hat{\mu}
$$

where $N_{m}$ is the number of monolayers and $\hat{\mu}$ is the magnetization unit vector in a given $\mathrm{Fe}$ layer. Such coupling would produce "spin" wave modes in a resonance experiment from which one could extract the dynamic coupling constant according to

$$
J=\frac{g \mu_{B} a\left(H_{u}-H_{s w}\right)}{8 d_{F e}}
$$

where $g$ is the spectroscopic splitting factor, $a$ is the Fe monolayer spacing, $d_{F e}$ is the $\mathrm{Fe}$ layer thickness and $H_{u}$ and $H_{s w}$ are the

Fig. 1 Raw FMR Spectra uniform and spin wave resonant fields respectively. This coupling constant arises from the highest frequency (optical) mode, and our $\mathrm{Fe}_{30} / \mathrm{Cr}_{18}$ data, shown in Fig. 2, supports this model in that two otherwise identical samples, which differ by factor of two in number of bilayers, yield the same J value. 


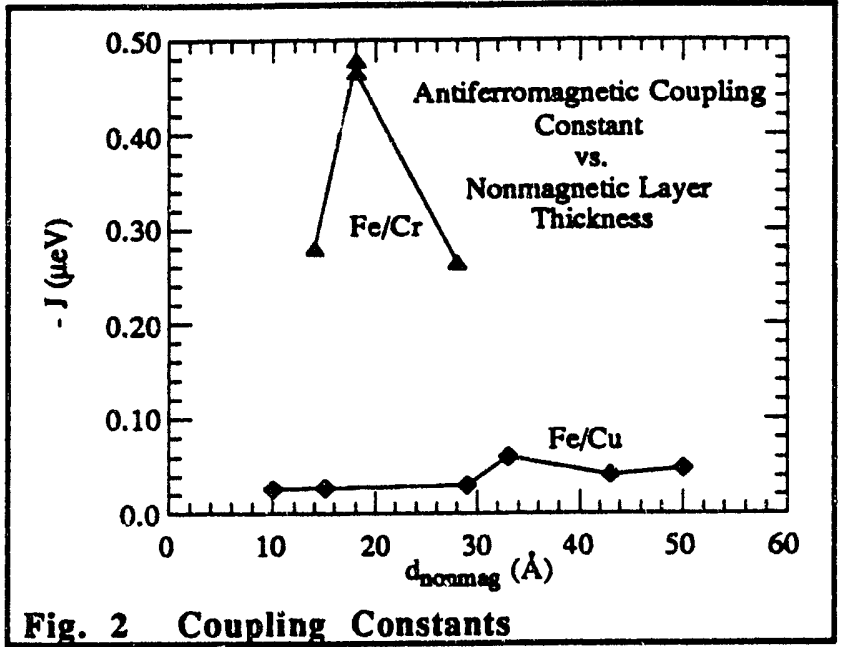

Although all coupling observed is antiferromagnetic, the dynamic coupling of $\mathrm{Fe}$ across $\mathrm{Cu}$ is an order of magnitude weaker than across $\mathrm{Cr}$. Such differences, consistent with static determination of the coupling strength, are manifest not only in the separation of the uniform and anomalous modes, but also their relative amplitudes - the interlayer precession phase shift being smaller in a strongly coupled system. Such characterization of dynamic coupling has been reported in trilayer systems ${ }^{1}$, however such characterization in the more complicated multilayers structures is, to my knowledge, novel in this investigation. As this report is being written, FMR measurements are revealing a very strong temperature dependence in the position and amplitude of the Fe/Mo anomalous modes, indicating interesting thermal properties of the interlayer coupling. We are in the process of extending our variable temperature FMR capability from the present $9 \mathrm{GHz}$ to $35 \mathrm{GHz}$ where the uniform and anomalous modes occur at higher fields ensuring domain saturation.

Several questions remain: What is the source of the interlayer coupling? Will the dynamic coupling exhibit the same oscillations with nonmagnetic layer thickness as observed by static means? Surprisingly, the uniform mode varies with nonmagnetic layer thickness, indicating modifications of the interface anisotropy. How are the interface anisotropy and interlayer coupling "coupled"? Can this coupling be engineered to produce desirable properties for applied material?

It is interesting to note that previously measured, Ni-based multilayer parallel FMR resonances show anomalous structure which we have, until recently, ignored. Closer scrutiny will be given these modes in view of interlayer coupling.

\section{Interface Anisotropy}

Our work on interface anisotropy (IA) has revealed an interesting contrast between $\mathrm{Ni} / \mathrm{V}$ and $\mathrm{Fe} / \mathrm{Cu}$ multilayers. Shown in Fig. 4 are the IA temperature dependences.

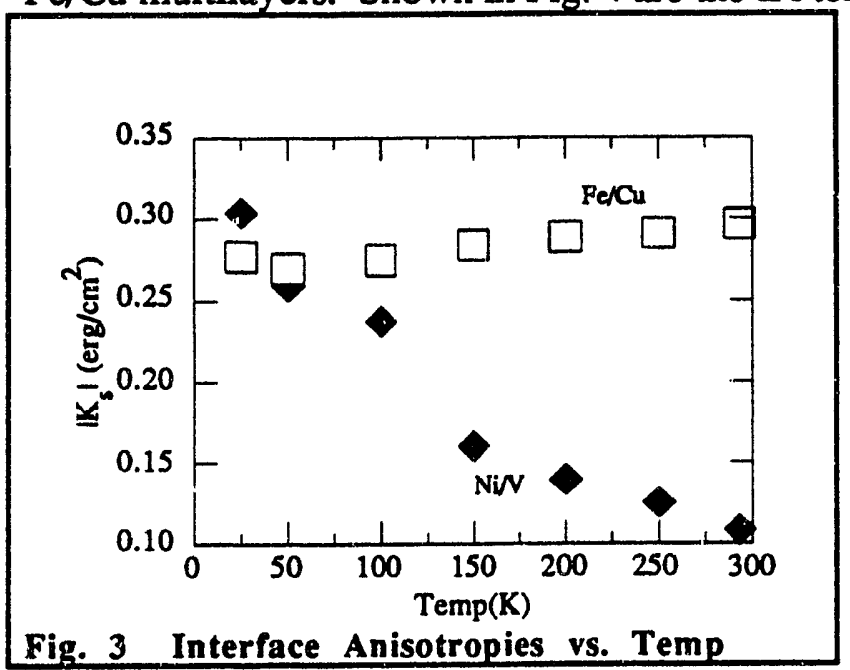

(Magnitudes are plotted because $\mathrm{Ni} / \mathrm{V}$ is inplane and $\mathrm{Fe} / \mathrm{Cu}$ is out-of-plane.) The IA in $\mathrm{Ni} / \mathrm{V}$ is approximately 30 times more strongly dependent upon temperature than that in $\mathrm{Fe} / \mathrm{Cu}$. This correlates strongly with the factor of 100 difference in the respective bulk $\mathrm{Ni}$ and $\mathrm{Fe}$ crystal field anisotropy temperature dependences, implying the IA source is closely tied to the interfacial crystal fields. It can be shown that the factor of 30 in the multilayers rather than 100 in the bulk is consistent with interfacial strain arising from $\mathrm{Ni} / \mathrm{V}$ lattice mismatch and magnetostriction. Due to the curious thermal behavior of volume anisotropy extracted from these measurements, publications of these results is pending measurements on new $\mathrm{Ni} / \mathrm{V}$ samples.

Techniques and Apparatus

Production of new Ni based multilayers has been delayed by two factors: 1) priority given to production of structures exhibiting interlayer coupling; and 2) establishment of Prof. Schuller's 
facilities at UCSD following his departure from Argonne. [The PI is presently (AY 92-93) on faculty improvement leave at UCSD to oversee production of the Ni multilayers.] This presented an opportunity to enhance our torque measurement and analysis techniques.

A novel, variable temperature, torque magnetometer based upon a commercially available transducer has been developed. ${ }^{2}$ The mechanism is based upon a differential capacitor whose low compliance provides high signal dynamic range [0.01 to 250 dyne-cm] and facilitates automation. Absolute calibration was accomplished with a current carrying loop, and the calibration constant is observed to be temperature independent to within $\pm 0.5 \%$ (see Fig. 5).

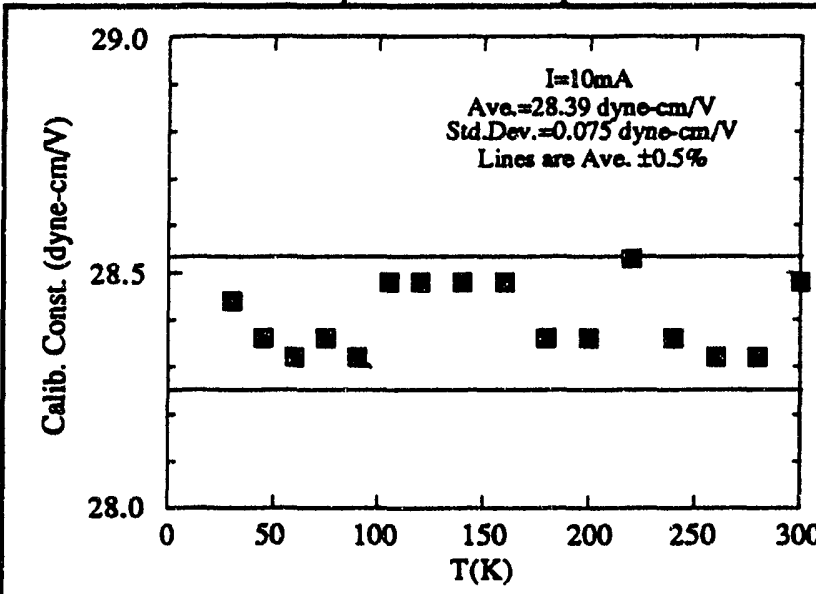

Fig. 4 Torque Calibration

Reliable operation below $60 \mathrm{~K}$ will require modification of the current magnetometer design, as occasional freezing of residual nonideal gases in the heat exchange system inhibit transmission of torque from the sample to the transducer. Modifications, such as locating a redesigned capacitive transducer at the site of the sample, are being tested.

We have developed numerical techniques for fitting torque uniaxial data which extracts the magnetization $(M)$ and first and second order anisotropies $\left(K_{1}\right.$ and $\left.K_{2}\right){ }^{3}$ The data is fit to the uniaxial model via a least squares method at many angles between the applied field and the "uniaxis", wherein each angle data set provides an independent measure of $M, K_{1}$ and $K_{2}$. The following difference function is minimized when the variables $m, k_{1}$ and $k_{2}$ are equal to $M, K_{1}$ and $K_{2}$ respectively, where $\tau_{i}$ and $H_{i}$ are data points taken at a constant angle $(\alpha)$ between the field and the uniaxis. $V$ is the magnetic volume.

$$
\begin{aligned}
& F\left(m, k_{1}, k_{2}\right)=\sum_{i=1}^{n}\left\{\tau_{i}-\left[\frac{2 k_{1} V}{\left(m V H_{i}\right)^{2}}\left(\sqrt{\left(m V H_{i}\right)^{2}-\tau_{i}^{2}} \sin \alpha-\tau_{i} \cos \alpha\right)\left(\sqrt{\left(m V H_{i}\right)^{2}-\tau_{i}^{2}} \cos \alpha-\tau_{i} \sin \alpha\right)\right.\right. \\
& \left.\left.+\frac{2 k_{2} V}{\left(m V H_{i}\right)^{4}}\left(\sqrt{\left(m V H_{i}\right)^{2}-\tau_{i}^{2}} \sin \alpha-\tau_{i} \cos \alpha\right)^{3}\left(\sqrt{\left(m V H_{i}\right)^{2}-\tau_{i}^{2}} \cos \alpha-\tau_{i} \sin \alpha\right)\right]\right\}^{2}
\end{aligned}
$$

During the analysis of our low-to-medium field torque data on $\mathrm{Fe} / \mathrm{Cu}$ multilayers, we realized that slight sample misalignment would introduce significant error in interpretation of the results. In order to quantify these effects, a series of measurements was made at known degrees of misalignment, and the results compared to theoretical expressions which contained explicitly the misalignment angle. Excellent agreement was obtained. A paper is being prepared which provides general quantification of such misalignment effects in torque uniaxial anisotropy determinations.

Our FMR research utilizes, almost exclusively, solid state microwave sources whose output frequency is automatically locked to the sample resonant cavity. The technology for providing such AFC to klystron tubes is not directly applicable to the low voltage solid state source and the variety of features found on a commercial lock-in amplifier are unnecessary for such control. Therefore we have developed a high performance, yet cost effective AFC based upon modern monolithic IC's, whose design and implementation has been published. ${ }^{4}$

\section{Superconductivity}

In order to concentrate the graduate student efforts primarily in magnetism, the PI's main area of expertise, the project's efforts in superconductivity are currently primarily limited to undergraduate research projects. This year an undergraduate student designed and constructed a thermal conductivity apparatus for measuring transport properties of $\mathrm{YBaCuO}$ and $\mathrm{BiCaSrCuO}$. 
Our results confirmed one of two conflicting results found in the literature on $\mathrm{YBaCuO}$ and agreed precisely with previous findings on $\mathrm{BiCaSrCuO}$. The student presented his results at The Argonne Symposium for Undergraduate Research at Argonne National Laboratory and submitted the results to The Journal of Undergraduate Research.

1J.J. Krebs, P. Lubiz, A. Chaiken and G.A. Prinz, Phys. Rev. Lett. 63, 1645 (1989). B. Heinrich, Z. Celinski, J.F. Cochran, W.B. Muir, J. Rudd, Q.M. Zhong, A.S. Arrott, K. Myrtle and J. Kirschner, Phys. Rev. Lett. 64, 673 (1990).

2 Michael J. Pechan, Michael E. Bait and Braciley D. Paul, Joum. Appl. Phys. 69, 5085 (1991). Variable temperature version submitted for publication.

${ }^{3}$ Bradley D. Paul and Michael J. Pechan, IEEE Trans. Magn. 27, 4846 (1991).

${ }^{4}$ Michael J. Pechan, Jiansheng Xu and Lynn D. Johnson, Rev. Sci. Instrum. 63, 3666 (1992). 


\section{DOE SUPPORTED PUBLICATIONS AND PRESENTATIONS (for the past year)}

\section{Publications in Print and Accepted}

"AFC Lock-in Amplifier For Magnetic Resonance." Michael J. Pechan, Jiansheng $\mathrm{Xu}^{*}$ and Lynn Johnson. Review of Scientific Instruments, 63, 3666 (1992).

"Generalized Torque Analysis of Magnetic Uniaxial Anisotropy." Bradley D. Paul* and Michael J. Pechan. IEEE Transactions on Magnetics, 27, 4846 (1991).

\section{Publications Submitted}

"Variable Temperature, Ultra-Low Compliance Torque Magnetometer." Michael J. Pechan, Alan P. Runge*, and Michael E. Bait*. Submitted to Review of Scientific Instruments.

\section{Publications in Preparation}

"Role of Nonmagnetic Layer in Transition Metal Multilayer Interface Anisotropy ", Michael J. Pechan, Zhong Lu and Ivan K. Schuller.

"Effects of Misalignment in Uniaxial Torque Measurements", Bradley D. Paul and Michael J. Pechan.

"Anomalous FMR Modes in Magnetic Multilayers - Evidence for Interlayer Coupling?" Michael J. Pechan, Jiangsheng Xu and Ivan K. Schuller.

\section{Presentations Made}

"Magnetic Multilayer Interface Physics and Interlayer Coupling". Physics Colloquium Presented at Wayne State University. April. 1992.

"Anomalous Parallel FMR Modes in $\mathrm{Fe} / \mathrm{Cu}$ and $\mathrm{Fe} / \mathrm{Cr}$ Multilayers: Evidence for Interlayer Coupling?". 1992 APS March Meeting. Indianapolis, IN.

"Magnetic Multilayer Interface Physics". Physics Colloquium Presented at Berea College. Feb. 1992.

"Dynamic Evidence for Interlayer Coupling in Fe Based Multilayers". Seminar Presented to Material Science Division at Argonne National Laboratory. Nov. 1991.

"Thermal Conductivity of High $\mathrm{T}_{\mathrm{c}}$ Superconducting Ceramics." James S. Middleton and Michael J. Pechan. Presented at the Symposium for Undergraduate Research, Argonne National Laboratory. Nov. 1991.

"Magnetic Multilayer Interface Physics". Physics Colloquium Presented at Denison University. Oct. 1991. 


\section{STUDENT INVOLVEMENT \\ (for the past year)}

\section{Masters Theses Graduated}

"Torque Magnetometry by Use of Capacitive-Type Transducer". Matthew Braught. August 1992.

"Variable Temperature Electron Spin Resonance Measurements of Plasticene". Charles Lynn. August 1992.

Masters Theses Presently Directing

"Temperature Dependence of Anomalous FMR. Modes in Fe/Mo Multilayers". JiangHong Huang. Projected August 1993.

\section{Undergraduate Student Participation}

(All students below are currently pursuing advanced degrees in Physics or Materials Science)

James Middleton. Started working on superconductivity as an incoming freshman. He set up a new furnace and controller for our laboratory and began making samples. His primary interests are in making the Bi-based compounds and measuring their thermal transport properties. James built a thermal conductivity apparatus and accurately measured the conductivities of $\mathrm{YBaCuO}$ and Bi-based sintered materials. He presented his results at a national conference and submitted the to the APS Journal for Undergraduate Research.

Wes Bailey and Scott McCall. Combined to redesign and construct a research grade superconducting magnet power supply.

Heather Frase. ESR measurements on $\mathrm{Mn}^{5 / 2}$ in modeling clay. Examining allowed and forbidden transitions in magnetic hyperfine structure. Went on make Raman measurements at Argonne under the Science and Engineering Research Semester program.

Malcolm Stanford. Worked on design and construction of gradient force magnetic particle suspension system. 

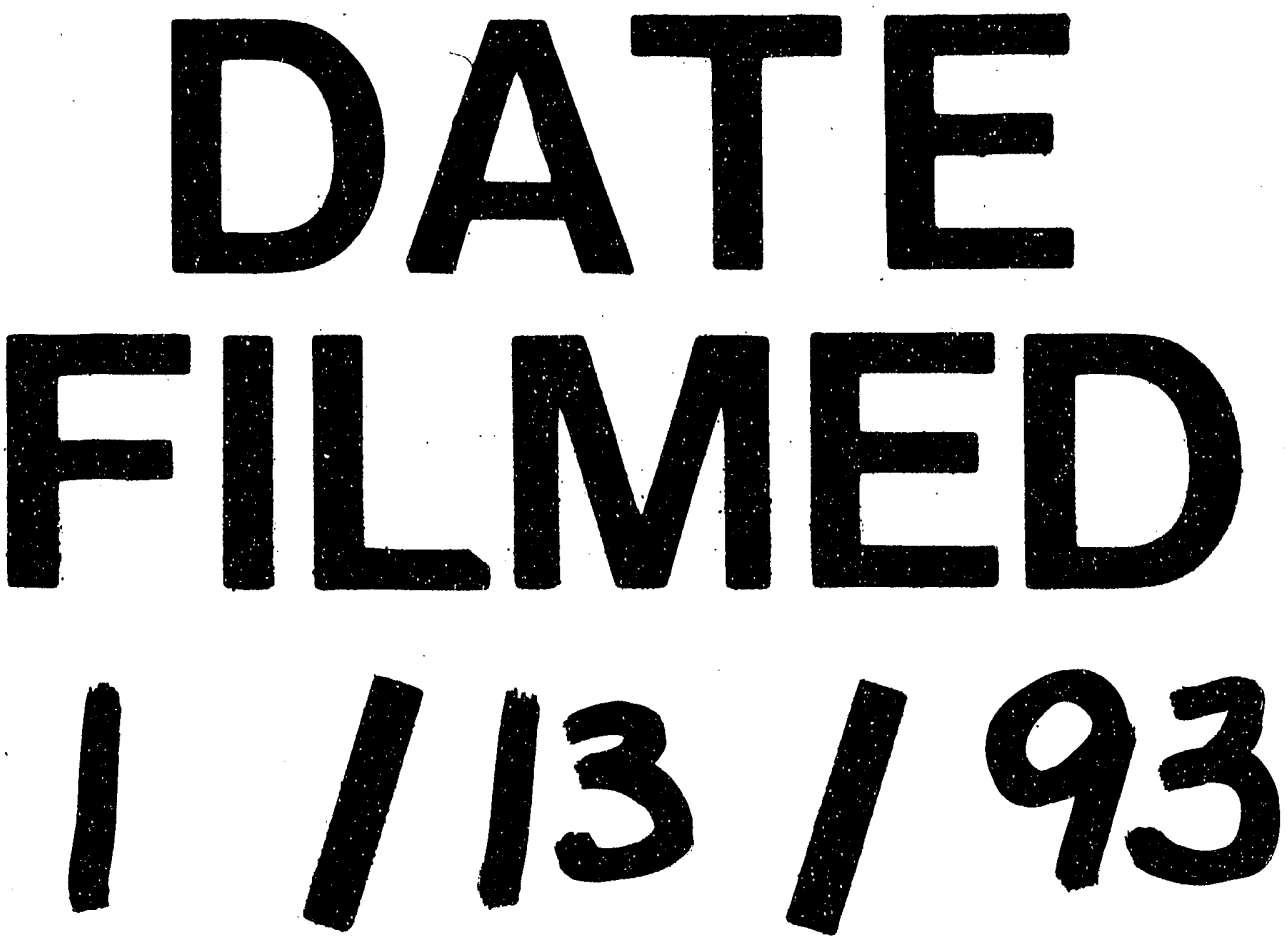
